(C) 2016 IEEE. Personal use of this material is permitted. Permission from IEEE must be obtained for all other uses, in any current or future media, including reprinting/republishing this material for advertising or promotional purposes, creating new collective works, for resale or redistribution to servers or lists, or reuse of any copyrighted component of this work in other works. 


\title{
SCEM+: Real-time Robust Simultaneous Catheter and Environment Modelling for Endovascular Navigation
}

\author{
Liang Zhao, Stamatia Giannarou, Su-Lin Lee and Guang-Zhong Yang, Fellow, IEEE
}

\begin{abstract}
Endovascular procedures are characterised by significant challenges mainly due to the complexity in catheter control and navigation. Real-time recovery of the 3D structure of the vasculature is necessary to visualise the interaction between the catheter and its surrounding environment to facilitate catheter manipulations. State-of-the-art intra-operative vessel reconstruction approaches are increasingly relying on non-ionising imaging techniques such as optical coherence tomography (OCT) and intravascular ultrasound (IVUS). To enable accurate recovery of vessel structures and to deal with sensing errors and abrupt catheter motions, this paper presents a robust and real-time vessel reconstruction scheme for endovascular navigation based on IVUS and electromagnetic (EM) tracking. It is formulated as a nonlinear optimisation problem, which considers the uncertainty in both the IVUS contour and the EM pose, as well as vessel morphology provided by pre-operative data. Detailed phantom validation is performed and the results demonstrate the potential clinical value of the technique.
\end{abstract}

Index Terms-Endovascular navigation, 3D vessel reconstruction, optimisation, intravascular ultrasound (IVUS), electromagnetic (EM) tracking.

\section{INTRODUCTION}

$\mathbf{E}$ NDOVASCULAR interventions are increasingly used for minimally invasive interventions to treat cardiovascular diseases [1]-[5]. These procedures induce minimal trauma to the patient but precise manipulation of catheters through the fragile and complex endovascular system in the presence of physiological motion remains a challenging task [6]-[8]. Although robotic systems have been introduced to facilitate catheter manipulations, they still rely on 2D guidance based on X-ray fluoroscopy and the use of contrast agents [9], [10]. The current clinical approach is to intra-operatively overlay 3D vessel models reconstructed from pre-operative imaging. However, due to the dynamic nature of the vasculature, these models should be updated in real-time, intra-operatively, to reflect the deformation of the vasculature. To establish safer endovascular procedures, knowledge of the interaction between the catheter and its surroundings is required and thus

Manuscript received: August 31, 2015; Revised November 25, 2015; Accepted January 16, 2016.

This paper was recommended for publication by Editor Ken Masamune upon evaluation of the Associate Editor and Reviewers' comments. This work was supported by the Commissions 7th Framework Programme FP7ICT Project "Cognitive AutonomouS CAtheter operating in Dynamic Environments (CASCADE), under grant agreement No.601021.

L. Zhao, S. Giannarou, S.-L. Lee and G. Z. Yang are with the Hamlyn Centre for Robotic Surgery, Imperial College London, London SW7 2AZ, United Kingdom. \{liang.zhao, stamatia.giannarou, su-lin.lee, g.z.yang\}eimperial.ac.uk

Digital Object Identifier (DOI): see top of this page. the 3D structure of the vasculature needs to be recovered intraoperatively.

Recently, concerns over ionising radiation and excessive use of nephrotoxic contrast agent have motivated the use of intravascular ultrasound (IVUS) for imaging guidance [11]. For vessel reconstruction, the main challenge associated with this technique is the limited field-of-view of cross-sectional data that is dependent on the pose of the transducer at the tip of the catheter. Initial approaches have utilised IVUS pullback data, whereby the image sequence is recorded as the catheter is slowly withdrawn from the vessel, but these assumed that IVUS images are parallel to each other [12], [13]. In practice, this is not realistic in areas where the curvature of the vessel is relatively large and the catheter moves abruptly during pullback, causing the 3D rotation and orientation of the catheter to change suddenly. The registration of IVUS images to angiography data to align the images in 3D space has been explored in [14]-[17] for vessel reconstruction but this still depends on X-ray imaging. Furthermore, the estimation of the $6 \mathrm{DoF}$ catheter pose in these approaches is not robust and additional sensing modalities are necessary to boost the accuracy of the vessel reconstruction. To this end, IVUS and electromagnetic (EM) sensing data have been fused for intraoperative vessel reconstruction in the SCEM framework [18], [19]. However, these existing methods treated observations from IVUS and EM as exact and their uncertainty was ignored. This makes the method vulnerable to errors in the observations which can affect the accuracy of the recovered vessel structure. For instance, vessel boundaries do not always appear complete on IVUS images and EM sensors are prone to measurement errors [20].

In this paper, a robust real-time $3 \mathrm{D}$ vessel reconstruction algorithm for endovascular catheter control and navigation (SCEM+) is proposed based on IVUS and EM sensing as shown in Fig. 1. To deal with the measurement errors from both EM sensors and IVUS images, the 3D vessel reconstruction is formulated as a nonlinear optimisation problem based on the pre-operative data. The uncertainty in both the IVUS contour and the EM pose are used as weights in the optimisation process. To enable vessel reconstruction in real-time, an approximation to the optimisation problem is proposed in which the first order linearisation related to solving the nonlinear optimisation can be computed a priori on the pre-operative data. Detailed validation on phantom datasets is performed to demonstrate the accuracy of the proposed algorithm and its robustness to abrupt catheter motions. 


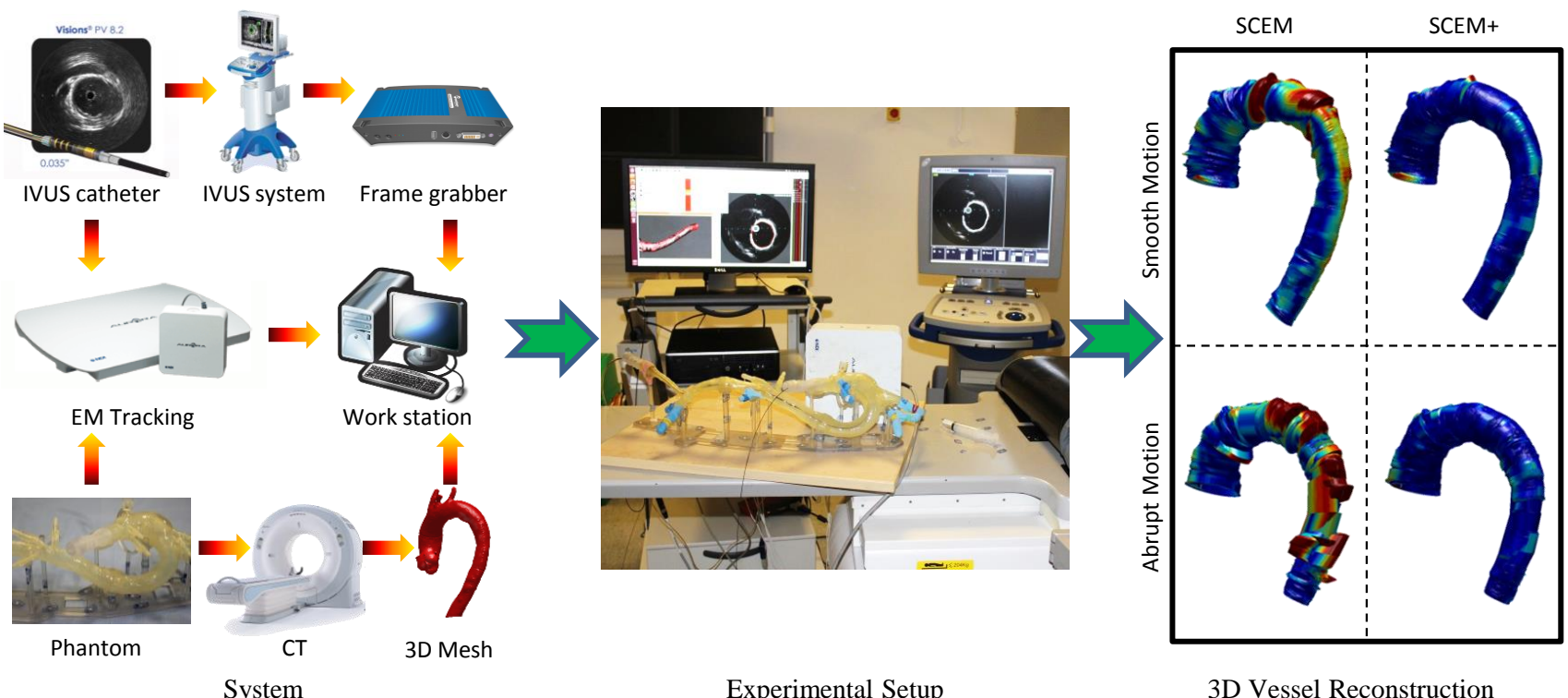

Fig. 1. The proposed SCEM+ framework: (left) the SCEM+ system components, (middle) experimental setup, and (right) the proposed improvements to the reconstruction.

\section{Vessel Modeling IN SCEM}

Simultaneous catheter and environment modeling (SCEM), as described in [18], proposes a reconstruction of the vasculature based on data fusion from intravascular ultrasound (IVUS) and electromagnetic (EM) tracking. In this framework, anatomical information is extracted by segmenting the contour of the inner vessel wall $C_{I}$ from the IVUS images which consists of a set of boundary points and is defined as:

$$
C_{I}=\left[c_{1}^{T}, \ldots, c_{m}^{T}\right]^{T}
$$

where each boundary point can be presented as

$$
c_{j}=\left[x_{j}, y_{j}, 0\right]^{T}, \quad j=1, \ldots, m .
$$

Here, the boundary point extracted from the IVUS image $\left[x_{j}, y_{j}\right]^{T}$ is padded with a 0 as the $\mathrm{z}$ coordinate to make $c_{j}$ a 3D point in the IVUS coordinate frame. The pose $\mathbf{P}_{E}$ captured by the EM sensor attached to the tip of the IVUS catheter is used to transform the IVUS contour $C_{I}$ to a global coordinate system by:

$$
C=\mathcal{R}_{E}^{T} C_{I}+\mathcal{T}_{E}
$$

where $\mathcal{R}_{E}$ and $\mathcal{T}_{E}$ can be computed as the Kronecker product:

$$
\begin{gathered}
\mathcal{R}_{E}=I_{m} \otimes R_{E}=\left[\begin{array}{ccc}
R_{E} & \cdots & 0 \\
\vdots & \ddots & \vdots \\
0 & \cdots & R_{E}
\end{array}\right] \\
\mathcal{T}_{E}=\mathbf{1}_{m} \otimes \mathbf{T}_{E}=\left[\mathbf{T}_{E}^{T}, \ldots, \mathbf{T}_{E}^{T}\right]^{T}
\end{gathered}
$$

where $R_{E}$ is the $3 \times 3$ rotation matrix and $\mathbf{T}_{E}$ is the $3 \times 1$ translation vector of the EM pose $\mathbf{P}_{E}, I_{m}$ is $m \times m$ identity matrix, $\mathbf{1}_{m}$ is $m \times 1$ vector with all elements equal to 1 and $\otimes$ is the Kronecker product. $C$ is the IVUS contour transformed into the global coordinate frame. Thus, the reconstruction of the vessel is obtained by transforming and combining all the IVUS contours extracted during vessel scanning to a common global coordinate system.

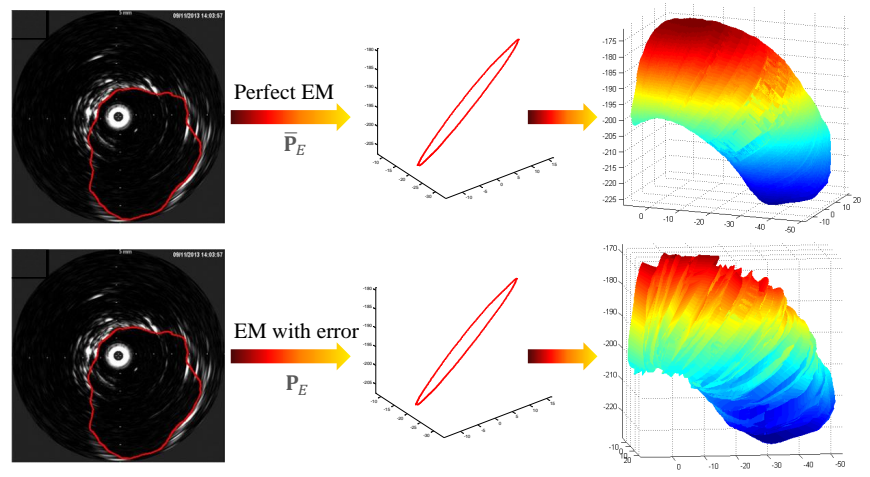

Fig. 2. The results from the original SCEM framework [18] show that the errors from IVUS/EM are directly transferred into the 3D vessel reconstruction.

However in SCEM, both the measurements from IVUS and EM are considered as exact, and any error associated with the measurements will transfer into the vessel reconstruction (see Fig. 2). The EM poses and IVUS contours are always associated with significant measurement errors which should not be ignored for accurate vessel reconstruction. For instance, the IVUS data is noisy and the vessel boundaries do not always appear complete on IVUS images, making the contour extraction challenging.

\section{METHODS}

In this paper, both the vessel contours from IVUS and the catheter poses from EM are considered as measurements with errors and uncertainties and are combined with the vessel structure provided by pre-operative data to formulate a nonlinear optimisation problem. The proposed vessel reconstruction framework and the improvements to the reconstruction are shown in Fig. 1. 


\section{A. Optimisation based on the Pre-operative Data}

The proposed nonlinear optimisation estimates the optimised catheter pose $\hat{\mathbf{P}}$ that will recover an accurate vessel structure. In the optimisation, the IVUS contour $C_{I}$ and the EM pose $\mathbf{P}_{E}$ are considered as observations with uncertainties defined by the covariance matrices $\Sigma_{I}$ and $\Sigma_{E}$ (to be discussed in Section III-B) respectively, and the catheter pose $\mathbf{P}=$ $[\alpha, \beta, \gamma, x, y, z]^{T}$ is defined as the state vector, where $[\alpha, \beta, \gamma]$ are the Z-Y-X Euler angles of the rotation and $\mathbf{T}=[x, y, z]^{T}$ is the translation vector. The nonlinear least squares problem can then be mathematically formulated as:

$$
\underset{\mathbf{P}}{\operatorname{argmin}}\left\|C_{I}-f(\mathbf{P})\right\|_{\Sigma_{I}^{-1}}^{2}+\left\|\mathbf{P}_{E}-\mathbf{P}\right\|_{\Sigma_{E}^{-1}}^{2}
$$

where

$$
\left\|C_{I}-f(\mathbf{P})\right\|_{\Sigma_{I}^{-1}}^{2}=\left[C_{I}-f(\mathbf{P})\right]^{T} \Sigma_{I}^{-1}\left[C_{I}-f(\mathbf{P})\right]
$$

denotes the weighted 2-norm of the residual vector of the IVUS contour $C_{I}-f(\mathbf{P})$ with the given covariance matrix $\Sigma_{I}$, where

$$
f(\mathbf{P})=\mathcal{R}\left(C_{T}-\mathcal{T}\right)
$$

and $\mathcal{R}$ and $\mathcal{T}$ are constructed similarly to (4) by using the rotation matrix $R$ and the translation vector $\mathbf{T}$ of the catheter pose $\mathbf{P}$. $R$ is computed from the Euler angles $[\alpha, \beta, \gamma]$. The contour $C_{T}$ is extracted from the pre-operative data as the cross section of the pre-operative CT model $M$ and the plane defined by the catheter pose $\mathbf{P}$. Here, $\mathbf{P}_{E}, C_{T}$ and the state vector $\mathbf{P}$ are all in the global coordinate frame while the IVUS contour $C_{I}$ is in the IVUS coordinate frame. The function $f(\cdot)$ transforms $C_{T}$ from the global into the IVUS coordinate system.

The first term in (5) aims to minimize the difference between the contour $C_{I}$ from the IVUS image and the contour $f(\mathbf{P})$ calculated from the pre-operative data, weighted by the uncertainty of the IVUS contour $\Sigma_{I}$. The second term in (5) minimizes the difference between the catheter pose $\mathbf{P}$ and the pose $\mathbf{P}_{E}$ obtained from EM, weighted by the uncertainty of the EM pose $\Sigma_{E}$.

A solution $\hat{\mathbf{P}}$ of (5) can be obtained with the Gauss-Newton method [21], starting with an initial estimate $\mathbf{P}_{0}$ and iterating with $\mathbf{P}_{k+1}=\mathbf{P}_{k}+\Delta_{k}$. The vector $\Delta_{k}$ is the solution to:

$$
\begin{aligned}
\left(J^{T} \Sigma_{I}^{-1} J+\Sigma_{E}^{-1}\right) \Delta_{k} & =J^{T} \Sigma_{I}^{-1}\left(C_{I}-f\left(\mathbf{P}_{k}\right)\right) \\
& +\Sigma_{E}^{-1}\left(\mathbf{P}_{E}-\mathbf{P}_{k}\right)
\end{aligned}
$$

where $J$ is the linear mapping represented by the Jacobian matrix $\partial f / \partial \mathbf{P}$ evaluated at $\mathbf{P}_{k}$. Here, $\mathbf{P}$ can be simply initialised by using the EM pose $\mathbf{P}_{0}=\mathbf{P}_{E}$. Given that $C_{T}(\mathbf{P})$ is not constant but a function of $\mathbf{P}$, the Jacobian $J$ can be computed by using the product rule for derivatives which first considers $C_{T}$ as constant, then considers $\mathcal{R}, \mathcal{T}$ in (7) as constant and only $C_{T}$ as a function of $\mathbf{P}$ as:

$$
J=\frac{\partial f_{C_{T}}}{\partial \mathbf{P}}+\mathcal{R} \frac{\partial C_{T}}{\partial \mathbf{P}}
$$

where $\partial f_{C_{T}} / \partial \mathbf{P}$ is the Jacobian with respect to $\mathbf{P}$ when considering $C_{T}$ as constant. Since the pre-operative CT model $M$ is discretized as triangle mesh, $C_{T}$ is not an analytic function. Thus, the Jacobian of $C_{T}$ with respect to the pose
$\mathbf{P}$ can be computed numerically on the pre-operative data by using the finite difference method [22]

$$
\frac{\partial C_{T}}{\partial \mathbf{P}} \approx \frac{C_{T}(\mathbf{P}+\Delta)-C_{T}(\mathbf{P}-\Delta)}{2 \Delta}
$$

where $\Delta$ is a small change on the state vector $\mathbf{P}$.

After an optimal solution of (5) is obtained, the uncertainty of the estimated catheter pose $\hat{\mathbf{P}}$ is given by the covariance matrix $\Sigma_{P}$ which can be computed by

$$
\Sigma_{P}^{-1}=J^{T} \Sigma_{I}^{-1} J+\Sigma_{E}^{-1} .
$$

Then, the 3D vessel shape can be recovered by transforming $C_{I}$ to the global coordinate frame using the optimised $\hat{\mathbf{P}}$, with reconstruction uncertainty $\Sigma_{C}$ :

$$
C=\hat{\mathcal{R}}^{T} C_{I}+\hat{\mathcal{T}}, \quad \Sigma_{C}=J_{C} \Sigma J_{C}^{T}
$$

where

$$
J_{C}=\left[\begin{array}{ll}
\frac{\partial C}{\partial \mathbf{P}} & \frac{\partial C}{\partial C_{I}}
\end{array}\right]
$$

is the Jacobian of $C$ with respect to the catheter pose $\mathbf{P}$ and IVUS contour $C_{I}$ respectively evaluated at $\hat{\mathbf{P}}$ and $C_{I}$, and

$$
\Sigma=\left[\begin{array}{ll}
\Sigma_{P} & \\
& \Sigma_{I}
\end{array}\right] .
$$

\section{B. Uncertainty Estimation}

To deal with the errors in the IVUS and EM measurements, the terms in the optimisation in (5) are weighted with the uncertainties $\Sigma_{I}$ and $\Sigma_{E}$. The uncertainty of the EM pose $\Sigma_{E}$ can be directly obtained from the position and orientation accuracy of the EM system. To estimate the uncertainty of the IVUS contour $\Sigma_{I}$, as the contour is represented as a set of boundary points $c_{j}, j=1, \ldots, m$, the covariance matrix of $C_{I}$ can be computed as:

$$
\Sigma_{I}=\left[\begin{array}{ccc}
\Sigma_{1} & \cdots & 0 \\
\vdots & \ddots & \vdots \\
0 & \cdots & \Sigma_{m}
\end{array}\right] \text {, where } \Sigma_{j}=\delta_{j}^{-1} I .
$$

where $I$ is the identity matrix. Here, the points of the contour are assumed to be independent, hence $\Sigma_{I}$ is block diagonal. The covariance matrix of each point $\Sigma_{j}$ is computed by the weight of the point $\delta_{j}$. The covariance matrix computed in this way is spherical.

In this paper, the intensities of the points around the extracted contour on the IVUS image are used to compute the weights $\delta_{j}$. The contour points $c_{j}$ are first transformed from the Cartesian coordinates to polar coordinates $\left(\rho_{j}, \phi_{j}\right)$ in the image coordinate frame. Afterwards, the intensities of all the image points on the direction $\phi_{j}$ within a distance $n$ from $\rho_{j}$ in the polar coordinate system are accumulated. The weight $\delta_{j}$ can be computed as:

$$
\delta_{j}= \begin{cases}1, & \text { if } \gamma_{j} \geq t \\ \gamma_{j} / t, & \text { if } \quad \gamma_{j}<t\end{cases}
$$

where

$$
\gamma_{j}=\sum_{l=0}^{n} V\left(\left(\rho_{j}+l\right) \cos \phi_{j},\left(\rho_{j}+l\right) \sin \phi_{j}\right) .
$$


Here $V$ is the IVUS image. According to (16), if $\gamma_{j}$ is greater than a threshold $t$, the point $c_{j}$ is considered to lie on the vessel wall and its weight is set to 1 . We assume that if a point has no high intensity neighbours, it does not belong to the vessel wall and its weight is set to 0 . An example of the estimation results of the uncertainty of the IVUS contour is shown in Fig. 9(b) and 9(c).

\section{Real-time Implementation}

To enable vessel reconstruction in real-time, the original objective function of the proposed optimisation scheme can be modified by left multiplying $\mathcal{R}^{T}$ to $C_{I}-f(\mathbf{P})$ in the first term in (5):

$$
\underset{\mathbf{P}}{\operatorname{argmin}}\left\|C_{T}-g(\mathbf{P})\right\|_{\Sigma_{I}^{-1}}^{2}+\left\|\mathbf{P}_{E}-\mathbf{P}\right\|_{\Sigma_{E}^{-1}}^{2}
$$

where

$$
g(\mathbf{P})=\mathcal{R}^{T} C_{I}+\mathcal{T}
$$

Here $C_{I}$ is transformed and compared with $C_{T}$ in the global coordinate frame. The above objective function is an approximation to our nonlinear optimisation (5) because the covariance matrix which should be used to weight the first term in (18) (which is the uncertainty of the IVUS contour in the global coordinates) cannot be directly computed since it requires the catheter pose $\mathbf{P}$ which is the state vector. For that reason, $\Sigma_{I}$ is used to approximate it.

The difference between the contours in (18) can also be represented as $\|\mathbf{d}\|^{2}=\left\|C_{T}-g(\mathbf{P})\right\|^{2}$. The distance vector $\mathbf{d}$ can alternatively be computed as the shortest distances from all the points on contour $g(\mathbf{P})$ to the CT model $M$ of the vessel in the pre-operative data. This can be pre-computed as the distance space $\mathcal{D}$ of the pre-operative data, which provides for each point $c$ in the global coordinate frame the shortest distance to the pre-operative mesh $M$ [23]

$$
\mathcal{D}(c)=\operatorname{sgn}(c) \min \|\operatorname{dist}(c, M)\|
$$

where

$$
\operatorname{sgn}(c)=\left\{\begin{aligned}
1, & \text { if } c \text { is inside } M \\
-1, & \text { if } c \text { is outside } M
\end{aligned}\right.
$$

And the corresponding Jacobian $J^{\prime}$ of $\mathbf{d}$ can then be computed as:

$$
J^{\prime}=-\frac{\partial \mathbf{d}}{\partial g} \frac{\partial g}{\partial \mathbf{P}} .
$$

Thus, the distance vector $\mathbf{d}$ can be obtained directly from the distance space $\mathcal{D}$, and its partial derivative can be computed as the gradient $\nabla \mathcal{D}$ of the distance space $\mathcal{D}$ :

$$
\mathbf{d}=\mathcal{D}(g(\mathbf{P})), \quad \frac{\partial \mathbf{d}}{\partial g}=\nabla \mathcal{D}(g(\mathbf{P})) .
$$

Here a signed distance space $\mathcal{D}$ is used instead of an unsigned one since otherwise the gradient will be incorrect if it is evaluated at the points which are close to the mesh surface.

The optimisation function formulated above is computationally efficient and enables real-time vessel reconstruction because the distance space $\mathcal{D}$ and the gradient $\nabla \mathcal{D}$ can be pre-calculated on the pre-operative data. This means there is no need to compute the Jacobian and the contour differences in

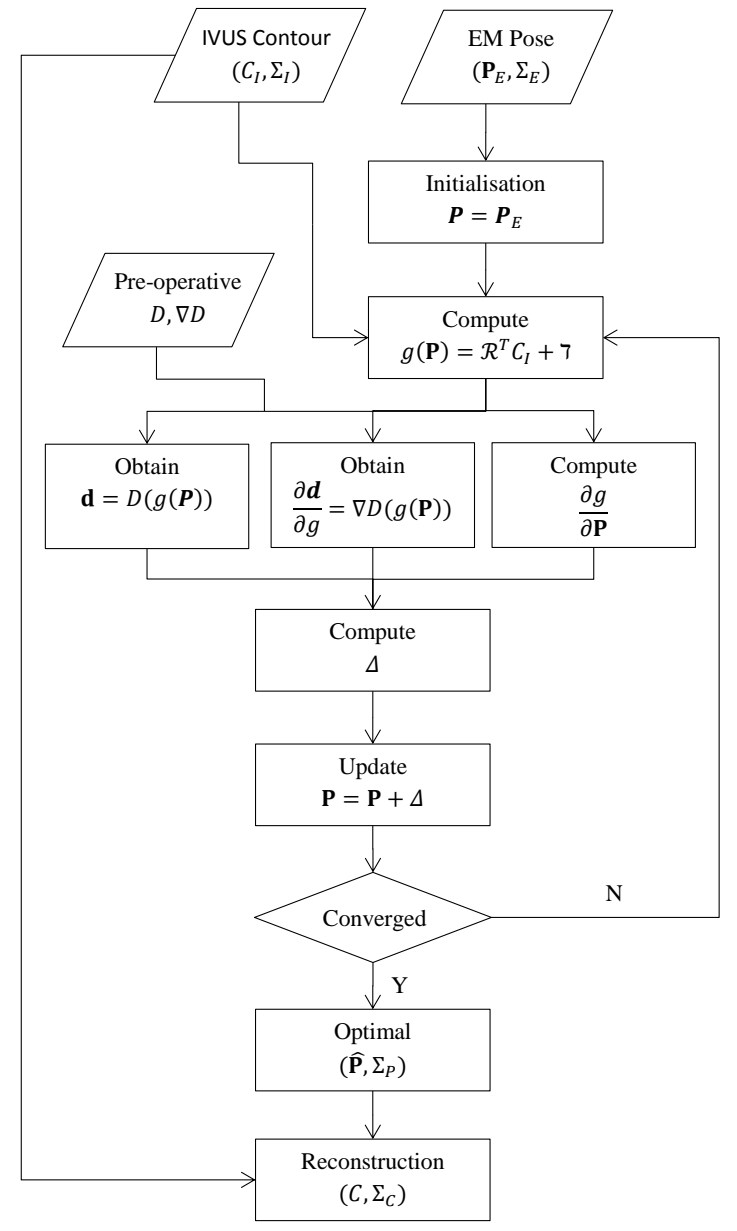

Fig. 3. A flowchart of the proposed real-time implementation of SCEM+.

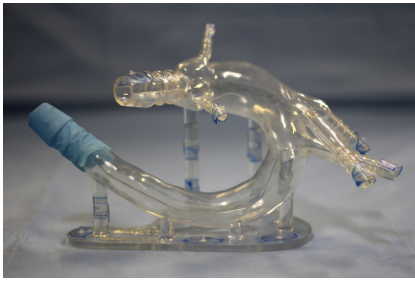

(a) Plexiglas Phantom

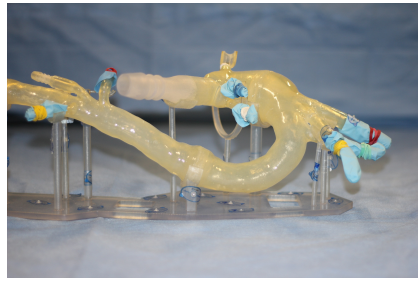

(b) HeartPrint Phantom
Fig. 4. The aortic phantoms used in the experiments: (a) Plexiglas and (b) HeartPrint aortic phantoms.

the first term of the objective function during the optimisation, allowing the reconstruction to be performed in real-time. The process of solving the problem by using the proposed realtime implementation is shown in the algorithm chart in Fig. 3.

To demonstrate the validity of the real-time implementation, here we give a lemma:

Lemma 1: The approximated problem in (18) is equivalent to the original problem in (5), if the covariance matrix of the IVUS contour $\Sigma_{I}$ is spherical.

Proof: The weighted 2-norm of the first term in (18) can be rewritten as

$$
\left\|C_{T}-g(\mathbf{P})\right\|_{\Sigma_{I}^{-1}}^{2}=\left[C_{I}-f(\mathbf{P})\right]^{T} \mathcal{R} \Sigma_{I}^{-1} \mathcal{R}^{T}\left[C_{I}-f(\mathbf{P})\right] .
$$



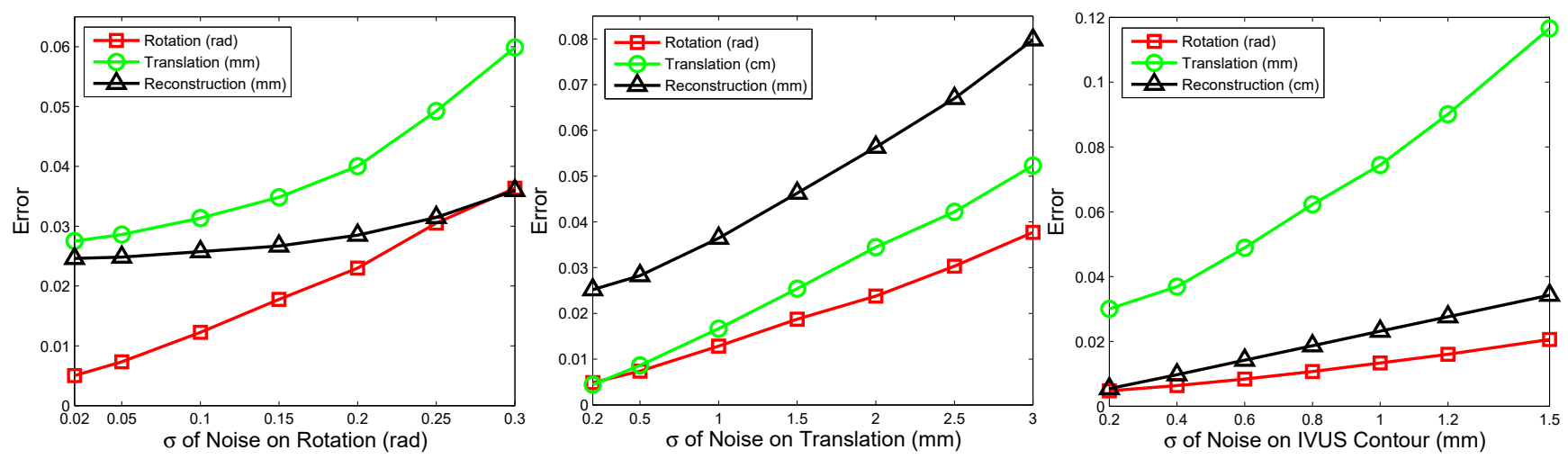

Fig. 5. The accuracy in the estimation of the catheter pose and 3D reconstruction w.r.t noise on the EM pose and extracted vessel contours.

If $\Sigma_{I}$ is spherical, $\mathcal{R} \Sigma_{I}^{-1} \mathcal{R}^{T}=\mathcal{R} \mathcal{R}^{T} \Sigma_{I}^{-1}=\Sigma_{I}^{-1}$ and (18) is equivalent to (5).

As described in Section III-B, since the covariance matrix $\Sigma_{I}$ is spherical, the approximated real-time implementation in (18) is equivalent to the original optimisation problem defined in Section III-A.

\section{RESUlts}

\section{A. Experimental Setup}

Two aortic phantoms created by Materialise (Leuven, Belgium) were used for validation, one made of plexiglas (Plexiglas) and the other made of HeartPrint ${ }^{\mathrm{R}}$ material (HeartPrint) (see Fig. 4). The two phantoms were first scanned by CT, and the meshes of the inner walls were segmented from the CTscan using ITK-SNAP [24] to provide the triangular surface meshes of the models (see Fig. 6). The distance space as well as the gradient of the distance space were pre-calculated from the 3D meshes. An Aurora 6DoF EM sensor (NDI, Waterloo, Canada) was attached to the tip of a Visions PV 8.2 IVUS catheter (Volcano, San Diego, USA) to provide its position and orientation.

The registration between the IVUS and EM sensors on the catheter was estimated by using a batch optimisation of the proposed algorithm for a pre-logged dataset, by considering all the catheter poses as well as the relative pose between the IVUS and EM sensors in the state vector. Since the IVUS-EM relative pose is fully correlated to all the catheter poses in the normal equations matrix, accurate results can be achieved. To register the CT and EM coordinate systems, CT markers (9 for Plexiglas and 13 for HeartPrint) attached to the phantoms were used to perform rigid registration.

\section{B. Simulation and Robustness Assessment}

Simulations were first performed to assess the influence of noise and robustness of the proposed algorithm to different levels of noise in the data. Simulated vessel contours were generated from one set of pre-operative data from the Plexiglas phantom along with known EM poses. Seven different levels of zero mean Gaussian noise were added to the EM poses and vessel contours and the results are shown in Fig. 5.

In this simulation, zero mean Gaussian noise with standard deviation $\sigma_{R}=\{0.02,0.05,0.1,0.15,0.2,0.25,0.3\} \mathrm{rad}$ was
TABLE I

DETAILS OF THE EXPERIMENTS

\begin{tabular}{lccccc}
\hline \hline Datasets & Date & Place* $^{*}$ & Phantom & Cath/Setup & Motion $^{\ddagger}$ \\
\hline $1-6$ & $06 / 2014$ & Hospital & Plexiglass & C-1/S-1 & Pul \\
$7-11$ & $12 / 2014$ & Hospital & HeartPrint & C-1/S-2 & Ins/Pul \\
$12-16$ & $01 / 2015$ & Hospital & HeartPrint & C-1/S-3 & Ins\&Pul+Abr \\
$17-23$ & $01 / 2015$ & KUL & HeartPrint & C-1/S-4 & Ins\&Pul \\
$24-25$ & $01 / 2015$ & KUL & HeartPrint & C-2/S-4 & Ins\&Pul \\
\hline \hline
\end{tabular}

*Hospital: Northwick Park Hospital, Imperial College London, KUL: University of Leuven. †C-1: Catheter 1, S-1: Setup-1. †Catheter motion: Pul(pullback), Ins(insertion) and Abr(abrupt catheter motion).

added to the rotation, $\sigma_{T}=\{0.2,0.5,1,1.5,2,2.5,3\} \mathrm{mm}$ to the translation of the EM poses, as well as $\sigma_{C}=$ $\{0.2,0.4,0.6,0.8,1.0,1.2,1.5\} \mathrm{mm}$ to the IVUS contours, and 10 independent runs of each noise level were generated to obtain a total of 210 datasets to validate the performance of the algorithm. For each level of noise, the mean errors of the 10 runs for rotation, translation and reconstruction of the results were computed and shown in Fig. 5 as red, green and black lines, respectively. Here, the maximum noise levels were within the range of the errors in the real EM and IVUS systems. The maximum noise level of $\sigma_{R}$ and $\sigma_{T}$ in the EM poses was defined as $0.3 \mathrm{rad}$ and $3 \mathrm{~mm}$, as the accuracy of the 6DoF sensor reported for the Aurora EM System was $0.8 \mathrm{~mm}(1.2 \mathrm{~mm} 95 \%$ confidence interval (CI)) for position and $0.012 \mathrm{rad}(0.014 \mathrm{rad} 95 \% \mathrm{CI})$ for orientation from the user guide. The maximum noise level of $\sigma_{C}$ was defined as $1.5 \mathrm{~mm}$ since the width of the high intensity vessel wall reflections in the IVUS images was around $1.5 \mathrm{~mm}$ which was measured from the IVUS images.

From Fig. 5 we can see that, increasing the noise in the rotation of the EM pose, the error in the estimation of the translation increased from $0.027 \mathrm{~mm}$ to $0.060 \mathrm{~mm}$ while the error of the 3D reconstruction only changed from $0.0246 \mathrm{~mm}$ to $0.0359 \mathrm{~mm}$. When adding noise to the translation of the EM pose, the accuracy of the translation was more sensitive than that of the rotation. Again, when adding noise to the IVUS contour, as noise increased, the error of the translation increased from $0.030 \mathrm{~mm}$ to $0.116 \mathrm{~mm}$, while the error of the rotation was not affected as much from $0.004 \mathrm{rad}$ to $0.021 \mathrm{rad}$. It can be seen that the accuracy of the catheter pose was still around $0.03 \mathrm{rad}$ and $0.12 \mathrm{~mm}$ even when noise of $0.3 \mathrm{rad}$ and $1.5 \mathrm{~mm}$ was added to the EM pose and the IVUS contours 


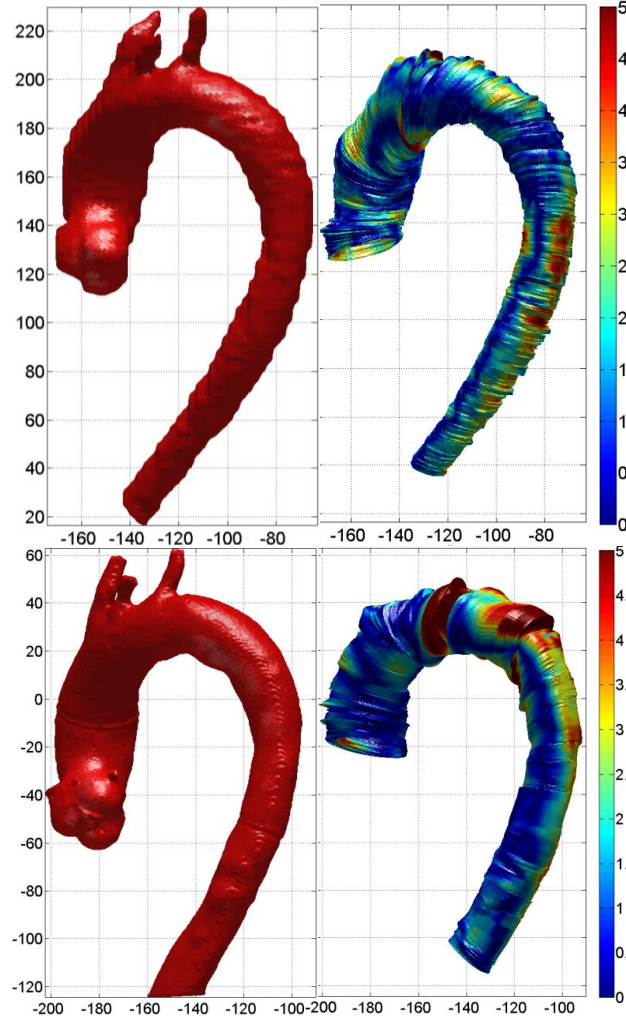

(a) 3D Mesh

(b) SCEM

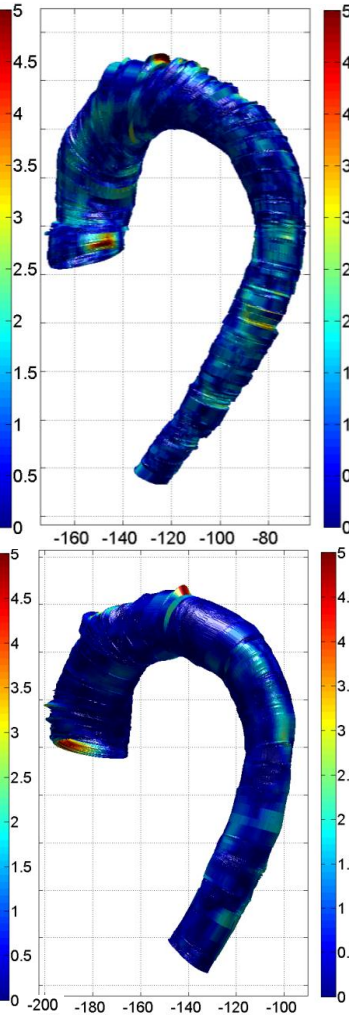

(c) $\mathrm{SCEM}+$

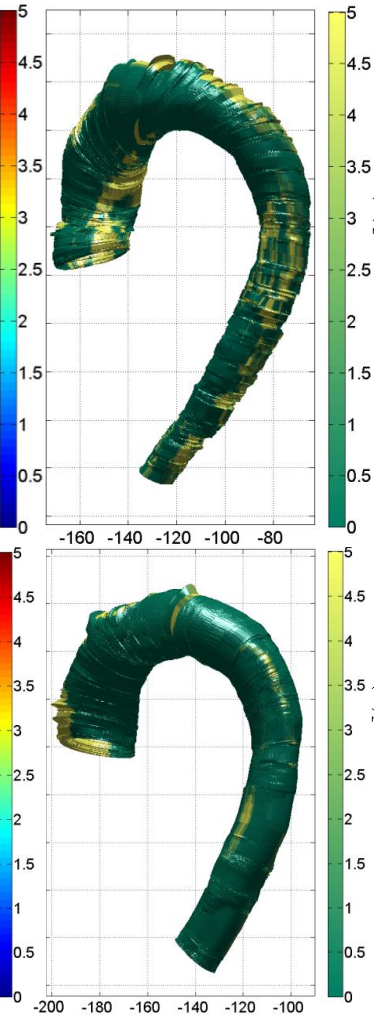

(d) Uncertainty

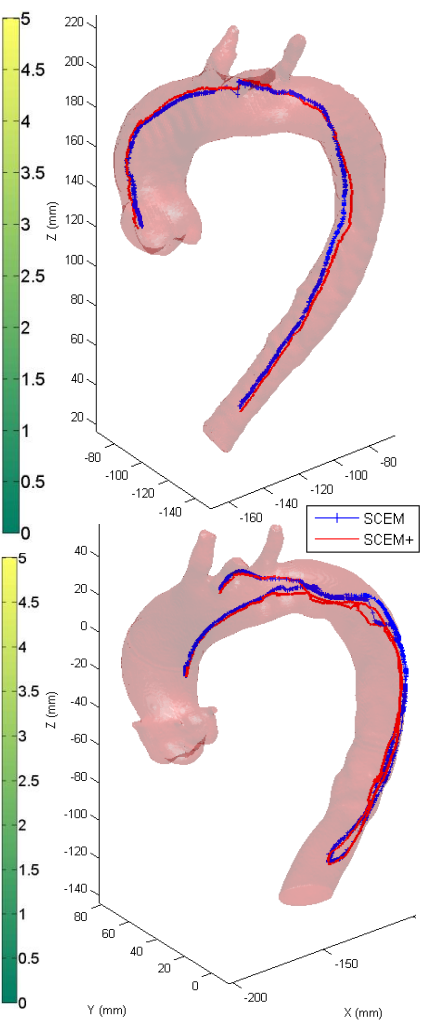

(e) Trajectories

Fig. 6. The results for the Plexiglas Phantom with pullback (first row, Dataset 3) and HeartPrint Phantom with insertion and pullback (second row, Dataset 21): (a) the 3D mesh of the pre-operative data, the 3D reconstruction results by (b) SCEM and (c) the proposed SCEM+ algorithm with errors in mm, (d) the uncertainty (log) map, and (e) the trajectories of the catheter computed by SCEM (blue) and SCEM+ (red).
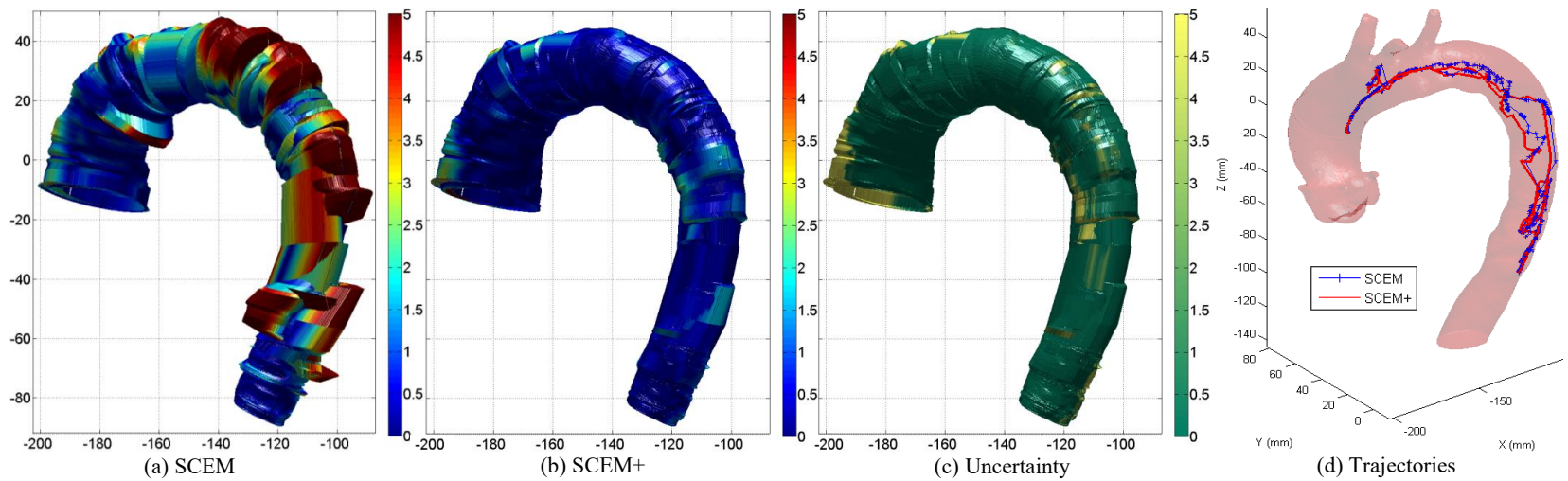

Fig. 7. The results for Dataset 14 in the HeartPrint phantom with abrupt catheter motion: (a) in the original SCEM algorithm, (b) in the proposed SCEM+ algorithm, (c) the uncertainty (log) map, and (d) the catheter trajectories.

respectively. The reconstruction accuracy was most sensitive when noise was added to the translation of the EM pose as well as to the IVUS contours.

\section{Phantom Experiments}

For the phantom experiments, a total of 25 datasets with 4 different EM-Phantom setups and 2 different IVUS-EM catheters were collected consisting of catheter pullbacks and/or insertions within the two phantoms. One of the setups is shown in Fig. 1. The details of the experiments are shown in Table I. In all the experiments, the catheters were not operated by medical expert.
For each IVUS image frame, contour extraction $(m=360)$ was performed with a radial scan similar to [18] to identify high intensity ultrasound reflections. To compute the uncertainty of the IVUS contour, $n=25$ pixels in the radial direction from each contour point in the polar coordinate frame are used. Here, 25 pixels were used in order to cover the maximum width of the reflections on the IVUS images which corresponds to the vessel wall. The performance of the proposed reconstruction on the two phantoms was compared to SCEM [18] in Fig. 6. The significant improvement over the use of SCEM in the presence of sudden catheter motion 


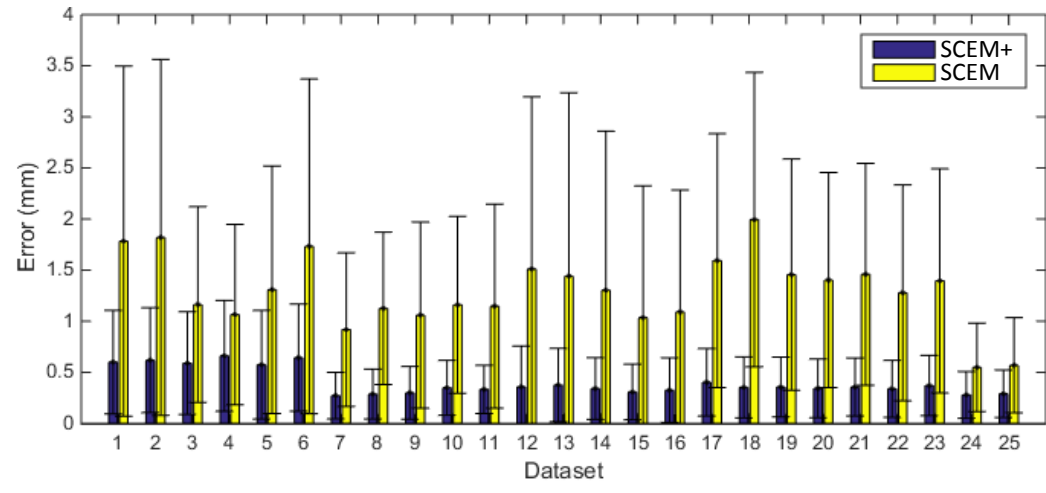

(a) Vessel Reconstruction Errors

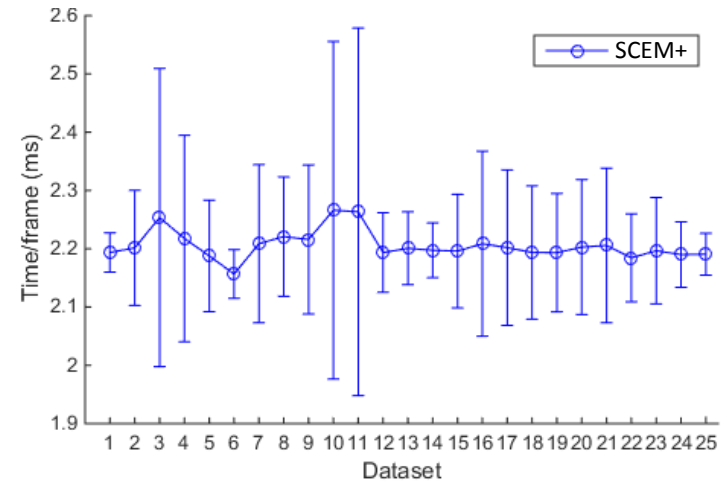

(b) Computational Cost

Fig. 8. The accuracy and computational cost of the proposed SCEM+ algorithm.

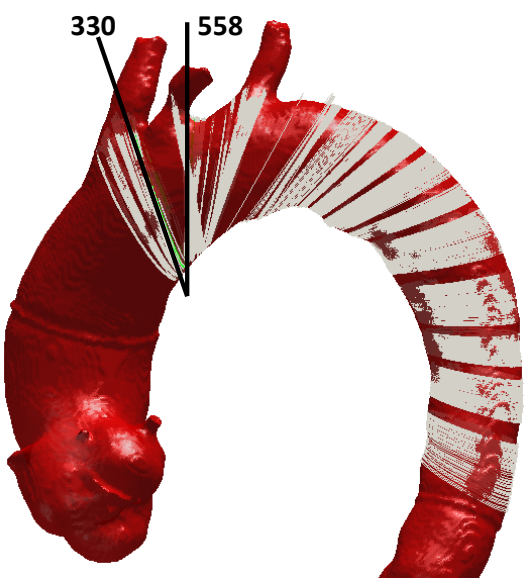

(a) 3D Contours

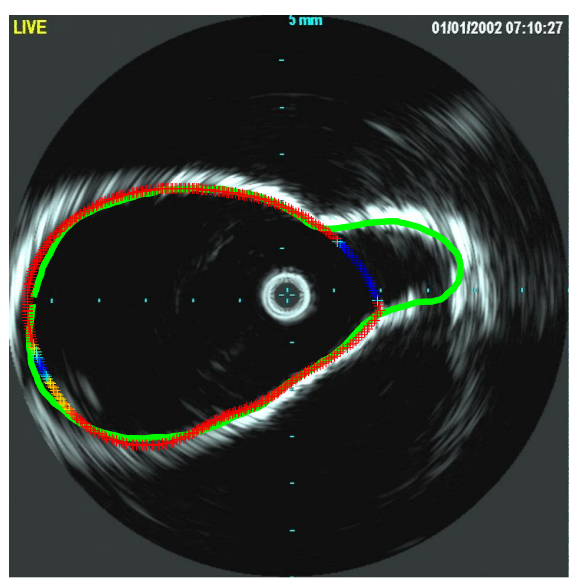

(b) IVUS Image 330

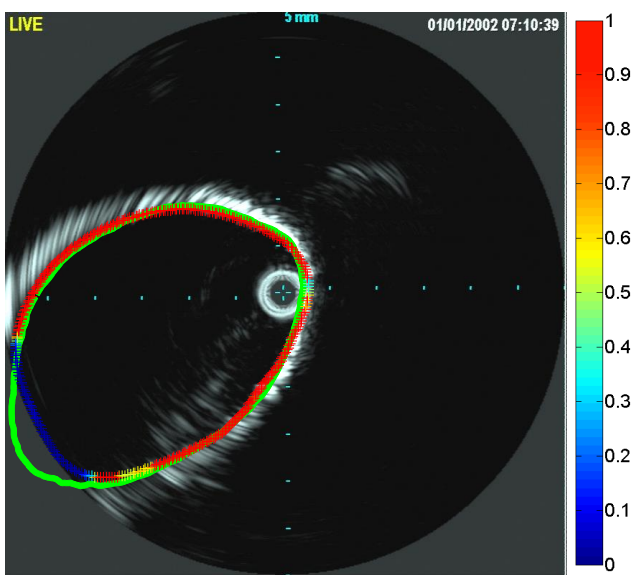

(c) IVUS Image 558

Fig. 9. Vessel contours back projected from the pre-operative mesh to IVUS images using the optimised poses from the proposed SCEM+ algorithm. The crosses represent the contour extracted from the IVUS images, coloured by the weight in (16) (between 0 to 1). The green line represents the back projected contours.

is highlighted in Fig. 7. The trajectories of the catheter tip computed by the two methods are shown in Fig. 6(e) and 7(d).

Quantitative evaluation of the performance of the proposed $\mathrm{SCEM}+$ algorithm is presented in Fig. 8(a). The reconstruction error was calculated as the distances between the recovered structure and the pre-operative data. The mean errors for the HeartPrint phantom are around $0.3 \mathrm{~mm}$, while the errors for the Plexiglas phantom are around $0.6 \mathrm{~mm}$, which are significantly lower than the errors of SCEM. The higher accuracy of the results for the HeartPrint phantom is due to the better quality of IVUS images within it compared to within the Plexiglas phantom due to their different material properties.

All the experiments were run on one core of an Intel i72600@3.4GHz CPU on a desktop. The computational cost of the optimisation algorithm presented in Fig. 8(b) verifies that the method can run in real-time. The algorithm converged in only 2-4 iterations with around $0.3-0.6 \mathrm{~ms}$ per iteration and the optimisation algorithm initialised by the pose from EM never diverged for any frame in the datasets.

To further validate the proposed vessel reconstruction, the optimised catheter poses from the proposed algorithm were used to obtain cross-sections of the pre-operative data which were back projected to the original IVUS images as shown in Fig. 9. It is clear that because of the consideration of the uncertainty, even with incomplete IVUS contour extraction, an accurate pose is still estimated as the corresponding vessel contour from the pre-operative model sits on the high intensity ultrasound reflections.

\section{CONCLUSION}

In conclusion, this paper presents SCEM+, a real-time robust $3 \mathrm{D}$ vessel reconstruction approach, which solves a nonlinear optimisation problem using IVUS, EM and preoperative data. Compared to other methods, the uncertainty of IVUS and EM were used as weights in the proposed optimisation algorithm to ensure that the reconstruction was robust to contour extraction error from IVUS and sudden motion of the catheter. It was also demonstrated that with the proposed reformulation of the objective function, a realtime implementation to the proposed optimisation scheme can be achieved. Simulation and experiments with two different phantoms demonstrated the efficiency and accuracy of the proposed algorithm against existing vessel reconstruction approaches. The method simultaneously obtains the vessel 
structure, optimal catheter pose, and the 3D reconstruction uncertainty which can be used for endovascular navigation.

This work is validated by phantom study. If in-vivo data is used, the global deformation can be recovered using EM sensors on the patient body and cardiac motion can be overcome using ECG gated IVUS. For our future work, to reconstruct all the deformation of the vessel, additional prior information will be included in the current objective function [25]-[27]. Finite element modelling [28]-[31] or differential surface representations [32]-[34] can also be combined with the current optimisation framework to provide boundary conditions for deformable vessels.

\section{REFERENCES}

[1] J. May, G. H. White, R. Waugh, P. Petrasek, X. Chaufour, M. Arulchelvam, M. S. Stephen and J. P. Harris, "Life-table analysis of primary and assisted success following endoluminal repair of abdominal aortic aneurysms: the role of supplementary endovascular intervention in improving outcome." European Journal of Vascular and Endovascular Surgery, vol. 19, no. 6, pp. 648-655, 2000.

[2] A. J. Avino, D. F. Bandyk, A. J. Gonsalves, B. L. Johnson, T. J. Black, B. R. Zwiebel, M. J. Rahaim and A. Cantor, "Surgical and endovascular intervention for infrainguinal vein graft stenosis." Journal of vascular surgery, vol. 29, no. 1, pp. 60-71, 1999.

[3] B. Funaki. "Endovascular intervention for the treatment of acute arterial gastrointestinal hemorrhage." Gastroenterology Clinics of North America, vol. 31, no. 3, pp. 701-713, 2002.

[4] V. Babaliaros and P. Block, "State of the art percutaneous intervention for the treatment of valvular heart disease: a review of the current technologies and ongoing research in the field of percutaneous valve replacement and repair." Cardiology, vol. 107, no. 2, pp. 87-96, 2007.

[5] A. Vahanian, O. R. Alfieri, N. Al-Attar, M. J. Antunes, J. Bax, B. Cormier, A. Cribier, P. D. Jaegere, G. Fournial, A. P. Kappetein and et al, "Transcatheter valve implantation for patients with aortic stenosis: a position statement from the European Association of CardioThoracic Surgery (EACTS) and the European Society of Cardiology (ESC), in collaboration with the European Association of Percutaneous Cardiovascular Interventions (EAPCI)." European Heart Journal, vol. 29, no. 11, pp. 1-8, 2008.

[6] T. Walther, M. W. Chu and F. W. Mohr, "Transcatheter aortic valve implantation: time to expand?" Current Opinion in Cardiology, vol. 23, no. 2, pp. 111-116, 2008.

[7] T. Walther, V. Falk, J. Kempfert, M. A. Borger, J. Fassl and M. W. Chu, "Transapical minimally invasive aortic valve implantation; the initial 50 patients." European Journal of Cardio-Thoracic Surgery, vol. 33, no. 6, pp. 983-988, 2008.

[8] R. Zahn, R. Schiele, C. Kilkowski, B. Klein, U. Zeymer, C. Werling, A. Lehmann, U. Gerckens and W. Saggau, "Correction of aortic regurgitation after transcatheter aortic valve implantation of the medtronic corevalvetm prosthesis due to a too-low implantation, using transcatheter repositioning." Journal of Heart Valve Disease, vol. 20, no. 1, pp. 64-69, 2011.

[9] M. J. Davidson, J. K. White and D. S. Baim, "Percutaneous therapies for valvular heart disease." Cardiovascular Pathology, vol. 15, pp. 123-129, 2006.

[10] J. Burgner, S. D. Herrell and R. Webster, "Toward fluoroscopic shape reconstruction for control of steerable medical devices." In Proc. ASME 2011 Dynamic Systems and Control Conference, pp. 791-794, 2011.

[11] L. W. Klein, D. L. Miller, S. Balter, W. Laskey, D. Haines, A. Norbash, M. A. Mauro, and J. A. Goldstein, "Occupational health hazards in the interventional laboratory: Time for a safer environment." Catheterization and Cardiovascular Interventions, vol. 73, no. 3 pp. 432-438, 2009.

[12] K. Rosenfield, D. W. Losordo, K. Ramaswamy, J. O. Pastore, R. E. Langevin, S. Razvi and J. M. Isner, "Three-dimensional reconstruction of human coronary and peripheral arteries from images recorded during two-dimensional intravascular ultrasound examination." Circulation, vol. 84, no. 5, pp. 1938-1956, 1991.

[13] R. Sanz-Requena, D. Moratal, D. R. Garca-Snchez, V. Bod, J. J. Rieta and J. M. Sanchis, "Automatic segmentation and 3D reconstruction of intravascular ultrasound images for a fast preliminary evaluation of vessel pathologies." Computerized Medical Imaging and Graphics, vol. 31, no. 2, pp. 71-80, 2007.
[14] A. Wahle, G. P. M. Prause, S. C. DeJong and M. Sonka, "Geometrically correct 3-D reconstruction of intravascular ultrasound images by fusion with bi-plane angiography-methods and validation." IEEE Transactions Medical Imaging, vol. 18, no. 8, pp. 686-699, 1999.

[15] B. Godbout, J. A. D. Guise, G. Soulez, and G. Cloutier, "3D elastic registration of vessel lumen from IVUS data on biplane angiography." In Proc. International Conference on Medical Image Computing and Computer Assisted Intervention (MICCAI), pp. 303-310, 2003.

[16] C. V. Bourantas, M. I. Papafaklis, L. Athanasiou, F. G. Kalatzis, K. K. Naka, P. K. Siogkas and L. K. Michalis, "A new methodology for accurate 3-dimensional coronary artery reconstruction using routine intravascular ultrasound and angiographic data: implications for widespread assessment of endothelial shear stress in humans." EuroIntervention, vol. 9, no. 5, pp. 582-93, 2013.

[17] C. V. Bourantas, I. C. Kourtis, M. E. Plissiti, D. I. Fotiadis, C. S. Katsouras, M. I. Papafaklis and L. K. Michalis, "A method for 3D reconstruction of coronary arteries using biplane angiography and intravascular ultrasound images." Computerized Medical Imaging and Graphics, vol. 29, pp. 597-606, 2005.

[18] C. Shi, S. Giannarou, S. L. Lee and G. Z. Yang, "Simultaneous catheter and environment modeling for Trans-catheter Aortic Valve Implantation.” In: Proc. IEEE/RSJ International Conference on Intelligent Robots and Systems (IROS), pp. 2024-2029, 2014.

[19] C. Shi, C. Tercero, S. Ikeda, K. Ooe, T. Fukuda, K. Komori and K. Yamamoto, "In vitro three-dimensional aortic vasculature modeling based on sensor fusion between intravascular ultrasound and magnetic tracker." International Journal of Medical Robotics and Computer Assisted Surgery, vol. 8, no. 3, pp. 291-299, 2012.

[20] A. D. Milne, D. G. Chess, J. A. Johnson, and G. J. W. King, "Accuracy of an electromagnetic tracking device: a study of the optimal operating range and metal interference." Journal of biomechanics vol. 29, no. 6, pp. 791-793, 1996.

[21] R. Hartley and A. Zisserman, Multiple view geometry in computer vision. Cambridge university press, 2003.

[22] K. W. Morton and D. F Mayers, Numerical solution of partial differential equations: an introduction. Cambridge university press, 2005.

[23] M. Jones, J. A. Baerentzen and M. Sramek, "3D distance fields: A survey of techniques and applications." IEEE Transactions on Visualization and Computer Graphics, vol. 12, no. 4, pp. 581-599, 2006.

[24] P. A. Yushkevich, J. Piven, H. C. Hazlett, R. G. Smith, S. Ho, J. C. Gee and G. Gerig, "User-guided 3D active contour segmentation of anatomical structures: Significantly improved efficiency and reliability." Neuroimage, vol. 31, no. 3, pp. 1116-28, 2006.

[25] L. Torresani, A. Hertzmann and C. Bregler, "Nonrigid structure-frommotion: Estimating shape and motion with hierarchical priors." IEEE Transactions on Pattern Analysis and Machine Intelligence, vol. 30, no. 5, pp. 878-892, 2008.

[26] Y. Dai, H. Li and M. He, "A simple prior-free method for non-rigid structure-from-motion factorization." International Journal of Computer Vision, vol. 107, no. 2, pp. 101-122, 2014.

[27] D. Terzopoulos, A. Witkin and Michael Kass, "Constraints on deformable models: Recovering 3D shape and nonrigid motion." Artificial Intelligence, vol. 36, no. 1, pp. 91-123, 1988.

[28] G. Celniker and D. Gossard, "Deformable curve and surface finiteelements for free-form shape design." In: Proc. ACM SIGGRAPH, pp. 257-266, 1991

[29] F. Cirak, M. Ortiz and P. Schroder, "Subdivision surfaces: A new paradigm for thin-shell finite-element analysis." International Journal for Numerical Methods in Engineering, vol. 47, no. 12, pp. 2039-2072, 2000.

[30] F. Cirak, M. Scott, P. Schroder, M. Ortiz and E. Antonsson, "Integrated modeling, finite-element analysis, and design for thin-shell structures using subdivision." $C A D$, vol. 34, no. 2, pp. 137-148, 2002.

[31] B. Thomaszewski, M. Wacker and W. Strasser, "A consistent bending model for cloth simulation with corotational subdivision finite elements." In: Proc. ACM SIGGRAPH/Eurographics Symposium on Computer Animation, pp. 107-116, 2006.

[32] M. Botsch and O. Sorkine, "On linear variational surface deformation methods." IEEE Transactions on Visualization and Computer Graphics, vol. 14, no. 1, pp. 213-230, 2008.

[33] M. Botsch, R. Sumner, M. Pauly and M. Gross, "Deformation transfer for detail-preserving surface editing." In: Proc. Vision, Modeling, and Visualization (VMV), pp. 357-364, 2006.

[34] Y. Yu, K. Zhou, D. Xu, X. Shi, H. Bao, B. Guo and H. Y. Shum, "Mesh editing with Poisson-based gradient field manipulation." $A C M$ Transactions on Graphics, vol. 23, no. 3, pp. 644-651, 2004. 